\title{
Nonequilibrium Quantum Transport Properties of Organic Molecules on Silicon
}

\author{
Wenchang Lu, ${ }^{1}$ Vincent Meunier, ${ }^{2}$ and J. Bernholc ${ }^{1,2}$ \\ ${ }^{1}$ Center for High Performance Simulation and Department of Physics, North Carolina State University, \\ Raleigh, North Carolina 27695-7518, USA \\ ${ }^{2}$ CSMD and CNMS, Oak Ridge National Laboratory, Oak Ridge, Tennessee 37831-6367, USA
}

(Received 5 April 2005; published 9 November 2005)

\begin{abstract}
Electron transport properties of a $\mathrm{Si} /$ organic-molecule/Si junction are investigated by large-scale nonequilibrium Green function calculations. The results provide a qualitative picture and quantitative understanding of the importance of self-consistent screening, broadening of quasimolecular orbitals under large bias, and enhancement of transmission, which occurs when the broadened lowest unoccupied molecular orbital aligns with the conduction band edge of the negative lead. The varying coupling can lead to negative differential resistance for a large class of small molecules.
\end{abstract}

PACS numbers: 73.63. $-\mathrm{b}, 71.15 .-\mathrm{m}, 73.40 . \mathrm{Gk}, 73.50 . \mathrm{Fq}$

Electron transport through molecular-scale devices has attracted considerable attention among both experimentalists and theorists. Most studies of these systems have focused on molecules connected to metallic leads [1-5]. Although some level of agreement between experiment and theory has been reached, several controversies remain, especially regarding the magnitude of the current, the mechanism of the negative differential resistance (NDR), the effect of junction geometry, etc. For example, for a very well-known molecular junction consisting of benzene-1,4dithiolate connected to gold electrodes, several theoretical calculations [4-6] reproduce the shape of the $I-V$ curve, while the conduction mechanism remains controversial. Using the jellium model to describe the metallic leads, Di Ventra et al. [4,5] found that the shape of the $I-V$ curve is mainly determined by the electronic structure of the molecule, while the atomic structure at the interface plays a key role in the magnitude of the current. They also found that the resonant tunneling occurs through the lowest unoccupied molecular orbital (LUMO), while Xue et al. [6] suggested that the electron transport occurs through filled states, i.e., the highest occupied molecular orbital (HOMO). Another calculation [7] is in closer agreement with experiment regarding the absolute value of the current, but did not reproduce the experimental shape of the $I-V$ curve. The reasons for these discrepancies might be the unknown structure of the contacts between the electrodes and the molecule and/or the different approximations made in the calculations of the current. Theoretical and experimental results for other molecules attached to metallic leads have been reviewed in Refs. [8,9].

Recently, several experimental and theoretical investigations of the transport properties of various organic molecules on Si surfaces have been reported [10-12]. These systems have a number of advantages compared to the traditional molecule-metal junctions. First, the organic molecules can be patterned on the Si surface [13,14], because of the thermodynamic and kinetic stability of the $\mathrm{Si}-\mathrm{C}$ bond. Second, the bonding between organic mole- cules and Si surface atoms is well understood [13-16], which should allow for straightforward comparisons between theoretical and experimental results on exactly the same atomic configuration. Third, there is a tremendous potential for applications combining $\mathrm{Si}$ microelectronics with organics-based structures; e.g., porphyrins on $\mathrm{Si}(001)$ are promising candidates for molecular memories [17]. Very recently, $I-V$ measurements for cyclopentene, 2,2,6,6-tetramethyl-1-piperidinyloxy (TEMPO), and styrene on $\mathrm{Si}(001)$ surfaces using a $\mathrm{Au}$ or $\mathrm{W}$ scanning tunneling microscope (STM) tips showed clear NDR features at room temperature [10,11], while suppression of NDR was observed for TEMPO and cyclopentene monolayers on $\mathrm{Si}(001)$. Datta and co-workers [12] investigated theoretically the conduction through styrene and TEMPO on $\mathrm{Si}$ using their hybrid-basis formalism (extended Hückel theory for Si and GAUSSIAN98 for the molecule, with the STM tip represented by a single tungsten atom). They found NDR effects due to molecular levels crossing the valence or conduction band edges, which cuts off transmission. They also found that the NDR positions vary with the tip-molecule distance.

In this Letter, we investigate the transport properties of 1,4-diethynylbenzene connected between two hydrogenated $\mathrm{Si}(111)$ surface fragments. In this paradigmatic geometry, the bonding to Si is well defined $[13,14]$, thereby avoiding the ambiguity of the contact geometry of metalconnected molecules. The calculations are carried out using the nonequilibrium Green function (NEGF) method $[18,19]$ in a basis of optimally localized orbitals [20,21]. Both the Si leads and the molecule are treated at the stateof-the-art density functional theory (DFT) level. The efficiency of the optimized orbital approach allows for the inclusion of more than 400 atoms in the "extended molecule," which fully accounts for the contact region and for the decay of the potential drop into the leads. We observe subtle effects, including significant broadening and selfconsistent shift of molecular levels with the applied voltage. The energy-dependent electron transmission not only 
shifts, but also significantly changes its shape as the voltage is increased, while the onset of negative differential resistance can still be interpreted in terms of changes of quasimolecular levels with bias and crossing of band edges. However, due to the presence of band gaps on each side of the molecule, the $I-V$ behavior is significantly more complex than in the Si-molecule-metal system. Furthermore, self-consistent screening becomes very important. In fact, while for the Si-molecule-metal system NDR was obtained in non-self-consistent calculations [12], a linear potential drop does not lead to NDR in the Simolecule-Si configuration and full self-consistency under bias is needed.

In our calculations, a unit cell with 12 layers of $\mathrm{Si}$ and 12 atoms per layer is used to describe each of the leads. Four localized, variationally optimized orbitals per atom are sufficient to obtain the total energy with an accuracy of $\sim 10 \mathrm{meV}$ per atom [22]. The conductor part used in constructing the Green function $G$ includes the molecule and 16 layers of $\mathrm{Si}$ on each side. Self-consistent iterations are carried out for each bias potential by the NEGF approach with complex energy contour integration [19]. The Hartree potential is obtained by solving the Poisson equation with boundary conditions corresponding to each bias; i.e., the potential in the conductor region is self-consistently matched on each side with the chemical potentials in the leads. Finally, the transmission coefficients are calculated from

$$
T(E, V)=\frac{2 e^{2}}{h} \operatorname{Tr}\left[\Gamma_{L}(E) G_{C}^{+}(E) \Gamma_{R}(E) G_{C}^{-}(E)\right]
$$

where $\Gamma_{L, R}$ and $G_{C}^{ \pm}$are the coupling functions for the left and right leads and the retarded and advanced Green functions of the conductor part, respectively. The current through the molecular junction is given by

$$
I(V)=\int_{-\infty}^{\infty} T(E, V)\left[f\left(E-\mu_{L}\right)-f\left(E-\mu_{R}\right)\right] d E,
$$

where $f$ is the Fermi-Dirac distribution and $\mu_{L, R}$ are the chemical potentials of the left and right leads, with $\mu_{L}-$ $\mu_{R}=V$ being the effective applied bias. The calculations assume intrinsic leads, with chemical potentials in the middle of the forbidden gap. When either or both of the semiconductor leads are doped, the chemical potential(s) will shift. In addition, surface termination often results in electron accumulation or depletion, which causes band bending and thus shifts in the positions of the band edges at the surface. However, both of these effects occur over the range of thousands of $\AA$. At the near-surface region relevant to ballistic transport, doping will thus manifest itself as a shift of the effective chemical potential measured with respect to the band edges. For example, if the left-lead region is $n$-type while the right one is $p$-type, the various $I-V$ features will appear at a lower bias, and vice versa.
As a test, the transmission though a pristine $\mathrm{Si}$ system is evaluated with the method described above. The results show quantized steplike transmission, as expected for a bulk system. The calculated conductance gap is $0.5 \mathrm{eV}$, which is due to the well-known underestimation of semiconductor band gaps by DFT. This result also confirms that our basis is complete enough for quantum transport calculations [23].

The atomic configuration of the 1,4-diethynylbenzene molecule on $\mathrm{Si}(111)$, optimized by total energy calculations, is shown in Fig. 1. One molecule per $(4 \times 3)$ unit cell is connected to the $\mathrm{Si}(111)$ surfaces through a single $\mathrm{Si}-\mathrm{C}$ bond, which is covalent with the typical bond length of $1.82 \AA$. The remaining dangling bonds of the Si surface are saturated by hydrogen atoms.

Two different ballistic transport calculations are performed with the applied bias, with and without selfconsistent iterations. In both cases, the charge density in the leads is fixed and the electrostatic potentials are shifted to match their chemical potentials. For the active region, the initial charge density is chosen from zero-bias DFT calculation and a linear potential drop across the conductor region is added to the electrostatic potential. In the nonSCF (non-self-consistent-field) calculations, the Hamiltonian matrix and the transmission coefficients are evaluated from the initial potentials. In the self-consistent calculations, the charge density and the potentials in the conductor region are determined iteratively, with the transmission coefficients obtained using the relaxed potentials and the updated matrix elements.

The transmission functions for the SCF calculations for biases of 0.0, 0.8, 1.4, and $2.0 \mathrm{~V}$ are shown in Fig. 2(a). The dashed lines represent the chemical potentials of the left and right leads. When the applied bias is smaller than the band gap of the bulk Si lead, the transmission in the bias window is zero. For $0.8 \mathrm{~V}$ bias, a small contribution originating mainly from the tail of the molecular LUMO appears in the bias window. With an increase of the bias,

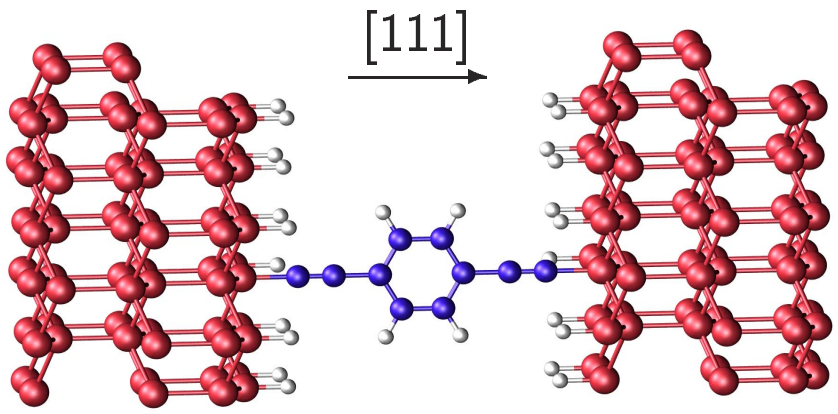

FIG. 1 (color online). Optimized geometry of the $\mathrm{Si}(111)-1,4-$ diethynylbenzene-Si(111) junction. The large (red), medium (blue), and small (gray) balls represent $\mathrm{Si}, \mathrm{C}$, and $\mathrm{H}$ atoms, respectively. The molecules are arranged periodically on the (111) surface with one molecule per $(4 \times 3)$ unit cell. Semiinfinite $\mathrm{Si}(111)$ on both sides of the molecule mimic the presence of the leads. 


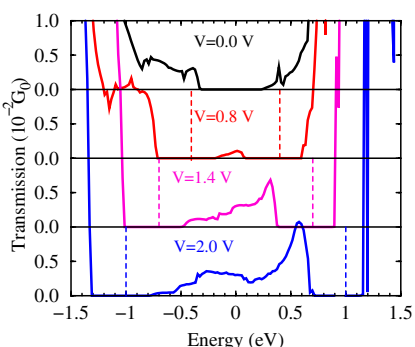

(a)

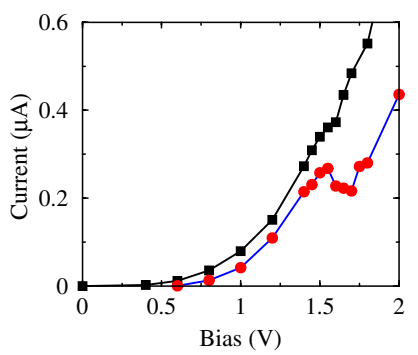

(b)
FIG. 2 (color online). (a) Self-consistent transmission coefficients through 1,4-diethynylbenzene connected to hydrogenated $\mathrm{Si}(111)$ leads. Bias windows are shown as dashed lines. The energy zeros are chosen to be at the center of each bias window. (b) $I-V$ curve for 1,4-diethynylbenzene between $\mathrm{Si}(111)$ leads. The squares and circles represent results from non-selfconsistent and self-consistent calculations, respectively. The lines are just a guide to the eye.

the molecular orbitals are broadened and the transmission increases. At $2.0 \mathrm{~V}$ bias, one can clearly see two transmission peaks in the bias window. One mostly arises from the tail of the LUMO and the other from the tail of the HOMO. Another interesting feature is the decrease of the transmission from the LUMO, when its center falls into the band gap of the left lead. This occurs at a bias of about $1.65 \mathrm{~V}$ and results in reduced coupling and thus in negative differential resistance, as described below.

The $I-V$ curves are calculated by integrating the transmission in the bias windows. They are shown in Fig. 2(b). From Fig. 2(b), it is clear that when the bias is smaller than $1.4 \mathrm{~V}$, the non-SCF calculations result in very similar $I-V$ characteristics to those of SCF calculations. In this bias region, the current exponentially increases with the applied bias, as expected for a system with a gap. However, when the bias is larger than $1.5 \mathrm{~V}$, self-consistency becomes very important. A very small shoulder appears at $1.6 \mathrm{~V}$ in the non-SCF calculation, while a current valley, i.e., a negative differential resistance, is observed in SCF calculations. This underlines the importance of performing fully selfconsistent calculations at large biases, when the device is far from equilibrium.

In order to understand the mechanism of electron transport through the molecule, we calculated the positiondependent density of states in the active region as

$$
\rho(\mathbf{r}, E)=-\frac{1}{\pi} \sum_{i, j} \phi_{i}^{*}(\mathbf{r}) \operatorname{Im}\left[G_{i j}^{+}(E)\right] \phi_{j}(\mathbf{r}),
$$

where $G$ denotes the Green function of the whole system under bias and $\phi_{i}(\mathbf{r})$ are the optimized localized orbitals. The density of states is averaged in the plane perpendicular to the current flow and color coded, with blue and red regions corresponding to low and high electron densities, respectively. The results for the biases of $0.0,0.8,1.4$, and 2.0 V are displayed in Fig. 3. The chemical potential at the right lead is chosen as the reference energy, which is different from Fig. 2(a).

Figure 3 clearly demonstrates the rearrangement of molecular levels with the applied bias. After the molecule is attached to the $\mathrm{Si}(111)$ leads and before any bias is applied, the gap between the HOMO and the LUMO is reduced to about $2.8 \mathrm{eV}$, a $0.2 \mathrm{eV}$ decrease compared to the isolated molecule. This is because the levels broaden slightly and the $\mathrm{C}-\mathrm{H}$ bond at each end of the molecule is replaced by a $\mathrm{C}$-Si bond. When the bias potential is increased, the levels become increasingly broader and their centers shift up relative to the chemical potential of the right lead. At a bias of $0.8 \mathrm{~V}$, the center of the LUMO is above the conduction band minimum, but its broadened tail (not visible on the scale of Fig. 3) contributes a small peak to the transmission [see Fig. 2(a)]. At a bias of about 1.4-1.5 V, the center of the LUMO reaches the conduction band edge of the left lead and a first peak in current is observed. With a further increase of the bias, the center of the LUMO falls into the band gap, weakening the hybridization between
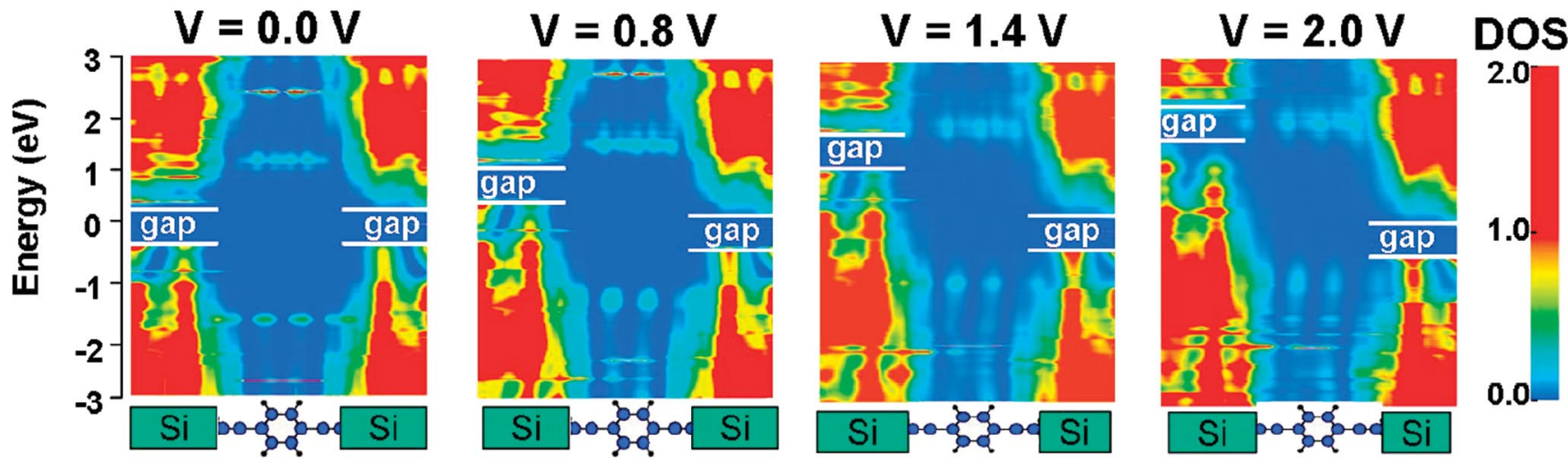

FIG. 3 (color). Position-dependent density of states (DOS) at biases of 0.0, 0.8, 1.4, and 2.0 V. The DOS is averaged in the plane perpendicular to the current. The blue and red colors represent regions of low and high density of states, respectively. The valence band maximum and conduction band minimum are indicated by white lines. The chemical potential of the leads lies in the middle of the band gap. 
the LUMO and the lead states. Correspondingly, the current decreases as the bias increases, marking the onset of NDR. For biases larger than $1.7 \mathrm{~V}$, the current increases again due to the following factors: First, the broadened HOMO tail results in the first peak in the transmission coefficient [at $-0.3 \mathrm{eV}$ in Fig. 2(a) for $V=2.0 \mathrm{~V}$ ]. Second, the valence bands of the left lead move up relative to the LUMO, overlapping its tail (not visible on the scale of the figure). This overlap contributes to another peak in the transmission coefficient [at $0.5 \mathrm{eV}$ in Fig. 2(a) for $V=$ $2.0 \mathrm{~V}]$. Clearly, the existence of valence and conduction band edges at both sides of the molecule results in biasinduced changes in electron transmission probabilities at each side of the molecule, and thus a complex, biasdependent shape of the transmission function. Nevertheless, negative differential resistance at one or more bias voltages is still to be expected, as the broadened molecular levels shift with the applied voltage. We should point out that due to the underestimation of band gaps and excitation energies by density functional theory, the experimental NDR will likely appear at larger biases than those predicted by the above calculations.

In summary, we have investigated quantum transport properties of a Si-molecule-Si system from first principles. Our results highlight the importance of self-consistent screening at large bias and the complex interplay between enhanced broadening of molecular levels and their rearrangement with respect to semiconductor band edges at both sides of the molecule. In the present system, enhanced tunneling occurs when the LUMO matches the conduction band edge of the negative lead, resulting in diminished coupling and in negative differential resistance when this orbital drops below the band edge. As the bias is further increased, tunneling occurs between the broadened HOMO and the valence band edge of the positive lead, resulting in a significant increase in transmission. The calculated position-dependent density of states for different biases clearly identifies the tunneling and broadening effects and provides a generic, broadly applicable picture of ballistic devices based on small molecules between semiconducting leads.

We would like to thank Dr. J. M. Buriak and Dr. P.T. Cummings for stimulating discussions at the initial stages of this project. This research was sponsored in part by U.S. Department of Energy under Contracts No. DE-FG0203ER46095 and No. DE-FG02-98ER45685, the DEAC05-00OR22725 contract with UT-Battelle, LLC at
Oak Ridge National Laboratory (ORNL), by the Laboratory Directed Research and Development Program of ORNL, and by the Office of Naval Research, under Contract No. N000149610161. The calculations were carried out at the DOD Supercomputing Centers.

[1] M. A. Reed, C. Zhou, C. J. Muller, T. P. Burgin, and J. M. Tour, Science 278, 252 (1997).

[2] A. Nitzan and M. A. Ratner, Science 300, 1384 (2003).

[3] J. Chen, M. A. Reed, A. M. Rawlett, and J.M. Tour, Science 286, 1550 (1999).

[4] M. Di Ventra, S. T. Pantelides, and N. D. Lang, Phys. Rev. Lett. 84, 979 (2000).

[5] M. Di Ventra, S. G. Kim, S. T. Pantelides, and N. D. Lang, Phys. Rev. Lett. 86, 288 (2001).

[6] Y. Xue, S. Datta, and M. A. Ratner, J. Chem. Phys. 115, 4292 (2001).

[7] P. Delaney and J. C. Greer, Phys. Rev. Lett. 93, 036805 (2004).

[8] J. Chen et al., Ann. N.Y. Acad. Sci. 960, 69 (2002).

[9] A. Pecchia and A. D. Carlo, Rep. Prog. Phys. 67, 1497 (2004).

[10] N.P. Guisinger, M.E. Greene, R. Basu, A. S. Baluch, and M. C. Hersam, Nano Lett. 4, 55 (2004).

[11] N. P. Guisinger, R. Basu, A. S. Baluch, and M. C. Hersam, Nanotechnology 15, S452 (2004).

[12] T. Rakshit, G. C. Liang, A. W. Ghosh, and S. Datta, Nano Lett. 4, 1803 (2004).

[13] R. J. Hamers, J. S. Hovis, S. Lee, H. Liu, and J. Shan, J. Phys. Chem. B 101, 1489 (1997).

[14] P. T. Hurley, A. E. Ribbe, and J. M. Buriak, J. Am. Chem. Soc. 125, 11334 (2003).

[15] W. C. Lu, W. G. Schmidt, and J. Bernholc, Phys. Rev. B 68, 115327 (2003).

[16] J. Cho and L. Kleinman, Phys. Rev. B 64, 235420 (2001).

[17] Z. Liu, A. A. Yasseri, J.S. Lindsey, and D.F. Bocian, Science 302, 1543 (2003).

[18] B. Larade, J. Taylor, H. Mehrez, and H. Guo, Phys. Rev. B 64, 075420 (2001).

[19] M. Brandbyge, J.L. Mozos, P. Ordejon, J. Taylor, and K. Stokbro, Phys. Rev. B 65, 165401 (2002).

[20] J.L. Fattebert and J. Bernholc, Phys. Rev. B 62, 1713 (2000).

[21] M. Buongiorno Nardelli, J.-L. Fattebert, and J. Bernholc, Phys. Rev. B 64, 245423 (2001).

[22] The orbital radii are $4.5 \AA$ and grid spacing is $0.13 \AA$.

[23] An insufficient basis can also result in unphysical spikes in the transmission coefficient. 